\section{GRP-149 PRESCRIBING ERRORS IN ANTINEOPLASTIC PRESCRIPTIONS}

doi:10.1136/ejhpharm-2013-000276.149

EY Romero Ventosa, M Rodriguez Rodriguez, S Gonzalez Costas, L Ezarte Lopez, A Mucientes Molina, K Lorenzo Lorenzo, N Lago Rivero, M Gayoso Rey, B Leboreiro Enriquez, G Piñeiro Corrales. Hospital Xeral-Cies de Vigo, Pharmacy, Vigo, Spain

Background Validation of antineoplastic prescriptions is an important job in hospital pharmacy to ensure appropriate patient treatment

Purpose To evaluate the prescribing errors in antineoplastic orders detected during oncology pharmacist validation.

Materials and Methods We conducted a two year prospective study (2010-2011) in which all prescriptions containing antineoplastic agents were reviewed for errors and all were accounted for in the analysis. Adjuvant medicines were excluded. One oncology pharmacist and one second year pharmacy resident were needed for this work. Prescriptions included: standardised chemotherapy order forms (SCOFs), individually typed and handwritten prescriptions. The primary outcome was the number of prescribing errors detected. The error rate was calculated by the ratio of the total number of prescription errors to the volume of prescriptions. Prescribing errors were then classified as follows: dose changed, antineoplastic error, dose reduction error, dose calculation error, dose omission, scheme changed, acronym changed, wrong patient identification, failure of therapeutic programme, antineoplastic omission and addition.

Results The number of prescribing errors detected was 80 . The error rate was $0.55 \%$ (for a total of 14,600 prescriptions). Principal types of errors detected were: dose changed (1\%), antineoplastic error $(5 \%)$, dose reduction error (14\%), dose calculation error $(32 \%)$, dose omission $(12 \%)$, scheme changed $(12 \%)$, acronym changed $(1 \%)$, wrong patient identification (1\%), failure of therapeutic programme (16\%), antineoplastic omission (5\%) and addition (1\%). None of the errors reached the patient.

Conclusions Our study points to the fact that, although chemotherapy prescribing errors are intercepted during pharmacist validation and do not reach the patient, there are still some problems in the chemotherapy ordering process and we should target preventive measures in order to improve patient safety.

No conflict of interest.

\section{GRP-150 PRESCRIPTION OF BISPHOSPHONATES IN CHRONICALLY INSTITUTIONALISED PATIENTS}

doi:10.1136/ejhpharm-2013-000276.150

'B Llagostera, 'M Hernandez, 'M Espier, ${ }^{2} \mathrm{C}$ Minguell. 'Mutuam, Prescription Quality Unit, Barcelona, Spain; ${ }^{2}$ Mutuam, Geriatric care teams EAR director, Barcelona, Spain

Background Osteoporosis is associated with significant morbidity and mortality. Oral bisphosphonates have become a mainstay of treatment, but concerns have emerged that long-term use of these drugs may suppress bone remodelling, leading to unusual fractures. Purpose To assess the intervention on bisphosphonates prescribing of institutionalised geriatric residential centres, by the Prescription Quality Unit (PQU).

Materials and Methods The PQU cheques that bisphosphonate treatment is based on patient age, duration of treatment, fracture, concomitant medicines and bisphosphonate prescribed. The PQU reviews the patients' medicines plans. The results of the review are communicated to the respective physicians, who analyse and discuss the medicines plans on the PQU report. The PQU performs regular clinical sessions and provides the doctors with drug data information (alerts, newsletters, surveys) involved in prescription reviews.
Results Interventions in bisphosphonates prescriptions from June 2011 to June 2012:

383 interventions were made (3.7\% of all interventions) and 86 were accepted, $22.4 \%$ on the bisphosphonates.

In 2011 one was accepted (19.11\%) while in 2012 27.2\% were accepted.

The mean age of patients with bisphosphonates was 86 years (10.63\% male and $89.37 \%$ female).

Bisphosphonates represented $4.1 \%$ of total prescriptions.

The breakdown of bisphosphonates prescriptions was $75.4 \%$ alendronate, $4.3 \%$ alendronate/cholecalciferol combination, $5.6 \%$ ibandronic acid and $14.61 \%$ risedronic acid.

Conclusions The intervention in bisphosphonates prescribing has been much more effective in 2012 than in 2011 and more intensive updates and drug date information has been provided to physicians in this period.

There were no problems in the use of the recommended bisphosphonate, alendronate.

No conflict of interest.

\section{GRP-151 PROGRAM INTERVENTIONS TO OPTIMISE THE DURATION OF ANTIBIOTIC TREATMENT}

doi:10.1136/ejhpharm-2013-000276.151

'P Aznarte, ${ }^{2} \mathrm{C}$ Hidalgo, 'M Ferrit, 'N Martínez, ${ }^{2} \mathrm{~J}$ Pasquau. ${ }^{1}$ University Hospital Virgen de las Nieves, Pharmacy, Granada, Spain; '2University Hospital Virgen de las Nieves, Infections Unit, Granada, Spain

Background Reducing unnecessarily prolonged antibiotic treatment is one of the main initiatives to ensure the appropriate use of antibiotics.

Purpose To analyse the preliminary results of a pharmaceutical interventions programme trying to minimise the duration of antibiotic treatment, promoted within the antibiotics stewardship programme of a tertiary hospital.

Materials and Methods A protocol was agreed on by the antibiotics stewardship group, to capture via the Pharmacy Department antimicrobial treatments lasting more than 10 days made through the electronic prescribing programme. A pharmacist intervened to suspend or change these antibiotics treatments. We excluded: oncohaematology patients, treatments for urinary tract infections, endocarditis, diabetic foot, empyema, if Pseudomonas aeruginosa was detected or when it was impossible to confirm the type of infection. We analysed the results of interventions from 27 January to 17 July 2012. The following variables were examined: antibiotics involved, prescriber department, number of interventions, acceptance, indication for treatment and treatment at discharge.

Results A total of 111 interventions were carried out. The most commonly used antibiotics were: amoxicillin/clavulanic acid (15.3\%), meropenem (13.5\%) and levofloxacin (13.5\%). The departments most involved were: Multipathological Care (43.2\%), Internal Medicine (35.1\%) and Pneumology (8.1\%). $74.8 \%$ of the antibiotic treatments were initiated because of respiratory infection. $18.9 \%$ of patients maintained antibiotics at discharge. The range of overall duration of antibiotic treatment in respiratory infection ranged from 10 to 20 days. The overall acceptance of the interventions was: $65.8 \%$. Within the accepted interventions, $68.5 \%$ caused antibiotic treatment to be suspended and $31.5 \%$ caused a change in the antibiotic used.

Conclusions The preliminary result of acceptance of interventions may be considered positive to ensure the programme is continued. To improve the acceptance of the interventions, it is necessary to increase involvement of Internal Medicine and Pneumology. The optimal duration of antibiotic therapy must assess the overall exposure, taking into account that established at the outpatient level.

No conflict of interest. 